\title{
Effective action of dilaton gravity as the classical double copy of Yang-Mills theory
}

\author{
Jan Plefka, ${ }^{1, *}$ Jan Steinhoff, ${ }^{2, \dagger}$ and Wadim Wormsbecher ${ }^{1,+}$ \\ ${ }^{1}$ Institut für Physik und IRIS Adlershof, Humboldt-Universität zu Berlin, \\ Zum Großen Windkanal 6, D-12489 Berlin, Germany \\ ${ }^{2}$ Max Planck Institute for Gravitational Physics (Albert Einstein Institute), \\ Am Mühlenberg 1, Potsdam D-14476, Germany
}

(Received 30 October 2018; published 14 January 2019)

\begin{abstract}
We compute the classical effective action of color charges moving along worldlines by integrating out the Yang-Mills gauge field to next-to-leading order in the coupling. An adapted version of the BernCarrasco-Johansson (BCJ) double-copy construction known from quantum scattering amplitudes is then applied to the Feynman integrands, yielding the prediction for the classical effective action of point masses in dilaton gravity. We check the validity of the result by independently constructing the effective action in dilaton gravity employing field redefinitions and gauge choices that greatly simplify the perturbative construction. Complete agreement is found at next-to-leading order. Finally, upon performing the postNewtonian expansion of our result, we find agreement with the corresponding action of scalar-tensor theories known from the literature. Our results represent a proof of concept for the classical double-copy construction of the gravitational effective action and provides another application of a BCJ-like double copy beyond scattering amplitudes.
\end{abstract}

DOI: 10.1103/PhysRevD.99.024021

\section{INTRODUCTION}

There is a growing body of evidence for a fascinating perturbative duality between Yang-Mills theory and quantum gravity known as the double-copy construction or color-kinematics duality due to Bern, Carrasco and Johansson (BCJ) [1-3]. It provides a concrete prescription for transforming scattering amplitudes in non-Abelian gauge theories into scattering amplitudes in gravitational theories upon replacing the non-Abelian color degrees of freedom by kinematical ones. In the simplest scenario pure Yang-Mills theory double copies to dilaton gravity coupled to an axion also termed as $\mathcal{N}=0$ supergravity (the massless sector of bosonic strings). This relation was proven for tree-level amplitudes [2], where the double copy is equivalent to the Kawai-Lewellen-Tye relations of string theory [4], a precursor of the BCJ duality. It is also manifest in the Cachazo-He-Yuan formulation of gluon and graviton tree-level amplitudes [5]. While remaining

\footnotetext{
*jan.plefka@physik.hu-berlin.de

jan.steinhoff@aei.mpg.de

wadim.wormsbecher@physik.hu-berlin.de
}

Published by the American Physical Society under the terms of the Creative Commons Attribution 4.0 International license. Further distribution of this work must maintain attribution to the author(s) and the published article's title, journal citation, and DOI. Funded by SCOAP ${ }^{3}$. conjectural at the loop level, the double-copy procedure is extremely efficient in generating integrands for gravitational theories at high-perturbative orders in theories with and without supersymmetry [6-14] and as such has passed many nontrivial checks. The present record being at the four point five-loop level for maximal supergravity [15]. Moreover, an elaborate web of theories connected via color-kinematics duality exists that includes matter couplings and various numbers of supersymmetries [16-18]. All these results point towards the double copy being a generic property of gravity alluding at a hidden kinematical algebra which has resisted discovery so far.

In light of these findings the natural question arises, whether the double copy generalizes beyond the realm of scattering amplitudes. In particular, does it also play a role in classical general relativity? Here a number of encouraging results have been obtained: Schwarzschild, Kerr and Taub-NUT spacetimes were shown to be double copies of classical gauge-theory solutions $[19,20]$, were further extended to certain classes of perturbative spacetimes [21], and a classical double-copy description of the gravitational radiation from accelerating black holes was developed in Ref. [22]. Following up the last direction, a very interesting setting is that of gravitational radiation produced by binary sources, not the least due to the spectacular observations of gravitational waves and the need for high precision predictions to generate waveform templates [23]. Pioneering work was done in [24-26] 
where perturbative classical solutions for binary colorcharged point particles coupled via Yang-Mills theory [27] were shown to double copy to their counterparts in dilatongravity radiation (see also [28,29]). In these works certain color-kinematics replacement rules were employed which appear somewhat distinct to the color factor/numerator replacement rules familiar from scattering amplitudes. Also the question of a double-copy-respecting representation of the perturbative gauge-theory solutions remained open. This situation was clarified very recently in the work of Shen [30], which pushed the perturbative approach of solving the equations of motions via double copy to the next-to-leading order (NLO) in the coupling constant expansion. In this process a color-kinematics duality representation of the Yang-Mills-radiation solution was found, which parallels the color-kinematics duality rules of BCJ [1] and replaces the nonstandard double-copy rules of [24]. An alternative route for finding the graviton radiation of binaries via the double copy was taken in [31], where a tree scattering amplitude in gauge theory coupled to scalar fields was double copied to establish the leading order gravitational radiation emitted from the scattering of two black holes (modeled by two massive scalar fields).

In our present work, we generalize these approaches to the double copy of gravitationally interacting binaries by ascending from the level of equations of motion to the classical effective action. This approach makes direct contact to the post-Minkowskian (weak-field) and postNewtonian (weak-field and slow-motion) expansions of the gravitational potential for which high-order results exist in the literature, namely at the fourth postNewtonian order (four loop) for nonspinning bodies: using a canonical formalism of general relativity [32], a Fokker Lagrangian [33,34], and partial results within an effective field theory formalism [35]. The latter makes use of Feynman diagrammatic methods and sophisticated tools for effective field theories in the context of classical gravity [36]. The question how the classical gravitational potential may be extracted from the quantum scattering amplitude of massive scalars has in fact a long history starting in the 1970s [37-39]. Recent works have updated this by employing modern unitarity methods of quantum field theory [40-42]. Damour recently proposed an alternative approach for converting scattering angles and amplitudes to the effective one-body Hamiltonian $[43,44]$. Hence, in order to perform the construction of the interaction potential using the double copy, one could study the scattering amplitude of a massive scalar field coupled to either YangMills or dilaton-gravity theory and relate to a potential using one of the mentioned works. Representing the gaugetheory result in a color-kinematics respecting fashion should then yield the effective gravitational potential via a suitably defined double copy. Here we follow a more direct approach, where the classical interaction potential is the direct outcome of a path integral. For this purpose, we start out with color charges moving along a worldline as in [24-26,30]. This may be viewed as a classical limit of the massive scalar field.

Our paper is organized as follows: In Sec. II we give an overview of the structure of a theory of classical colorcharged massive point particles moving along worldlines, interacting via a Yang-Mills theory, in the first-order formalism. In Sec. III we propose a double-copy prescription for the classical effective action. We compute the effective action of the theory introduced in Sec. II up to next-to-leading order in the gluon coupling. Along the way we introduce the notion of a trivalent representation for graphs involving worldlines and at the end of the section we perform the double copy. In Sec. IV we repeat the computation of the effective action in the weak-field/postMinkowskian approximation for massive point particles interacting in dilaton-gravity theory and we compare the resulting expression to the double-copy result. In Sec. V we reexpand our result for the post-Minkowskian dilatongravity effective action in velocities, thus yielding the postNewtonian approximation. After solving the Feynman integrals, we compare the resulting expression to known results in the literature. Our conclusions are presented in Sec. VI. Finally, our conventions, the Feynman rules and a discussion of self-interactions can be found in the appendices.

\section{YANG-MILLS INTERACTION OF COLOR-CHARGED MASSIVE PARTICLES}

We consider the worldline action of a massive, colorcharged (non-Abelian) point particle coupled to the YangMills gauge field $A_{\mu}^{a}$; see appendix A for notation and conventions. The action for the classical colored point charge (pc) is given by $[24,25,27]$

$$
\begin{aligned}
S_{\mathrm{pc}} & =-\int d \tau L_{\mathrm{pc}}=-\int d \tau\left(m \sqrt{u^{2}}-\psi^{\dagger} i u^{\mu} D_{\mu} \psi\right) \\
& =-\int d \tau\left(m \sqrt{u^{2}}-i \psi^{\dagger} \dot{\psi}-g u^{\mu} A_{\mu}^{a} c^{a}\right),
\end{aligned}
$$

which is invariant under reparametrization of $\tau$. Here $u^{\mu}=$ $\dot{x}^{\mu}$ is the 4-velocity of the particle along the worldline $x^{\mu}(\tau)$ and $\psi(\tau)$ an associated fundamental vector carrying the color degrees of freedom of the particle-often called the color wave function. Moreover

$$
c^{a}:=\psi^{\dagger} T^{a} \psi
$$

is the color charge carried by the particle. In this form, there is only a single gluon coupling to the scalar worldline. As the gravity counterpart also has higher graviton couplings to the worldline of a massive particle, this poses an immediate obstacle to a double-copy relation. Also a massive scalar field, for which a double copy of scattering 
amplitudes exist [31], has linear and quadratic couplings to gluons. However, in a first-order formalism this situation is remedied. Defining canonical momenta

$$
p_{\mu}=\frac{\partial L_{\mathrm{pc}}}{\partial u^{\mu}}=m \frac{u_{\mu}}{\sqrt{u^{2}}}-g A_{\mu}^{a} c^{a}, \Rightarrow m^{2}=(p+g A c)^{2},
$$

we find the Hamiltonian and the action in the first-order formalism

$$
\begin{gathered}
H_{\mathrm{pc}}=H_{\mathrm{can}}+\lambda\left[(p+g A c)^{2}-m^{2}\right], \\
H_{\mathrm{can}}=p_{\mu} u^{\mu}-L_{p c}{ }^{(4)}=i \psi^{\dagger} \dot{\psi}, \\
S_{\mathrm{pc}}=-\int d \tau\left(p_{\mu} u^{\mu}-H_{\mathrm{pc}}\right) \\
=-\int d \tau\left(p_{\mu} u^{\mu}-i \psi^{\dagger} \dot{\psi}-\lambda\left[p^{2}+2 g p_{\mu} A_{a}^{\mu} c^{a}\right.\right. \\
\left.\left.+g^{2} A_{\mu}^{b} c^{b} A_{a}^{\mu} c^{a}-m^{2}\right]\right) .
\end{gathered}
$$

Here, $H_{\text {can }}$ is the "canonical" Hamiltonian and $H_{\mathrm{pc}}$ is the "Dirac" Hamiltonian where the mass-shell constraint was added using a Lagrange multiplier $\lambda(\tau)$. Now we have both a single and a double gluon coupling to the worldline, similar to a massive scalar field. Note that $\lambda(\tau) d \tau$ is reparametrization invariant.

We now consider Yang-Mills gauge theory coupled to a set of two scalar particles in the worldline description. Notationally we separate the worldlines by placing a tilde on all variables associated to one of the worldlines. Therefore the full action for classical Yang-Mills (cYM) reads

$$
S_{\mathrm{cYM}}=S_{\mathrm{YM}}+S_{\mathrm{gf}}+S_{\mathrm{pc}}+\tilde{S}_{\mathrm{pc}},
$$

where a gauge fixing part $S_{\mathrm{gf}}$ was added. With this, the gluon propagator reads

$$
\left\langle A_{\mu}^{a}(x) A_{\nu}^{b}(y)\right\rangle_{0}=\frac{\hbar}{i} \eta_{\mu \nu} \delta_{a b} D(x-y),
$$

where $D(x-y)$ is defined via

$$
\square D(x-y)=-\delta(x-y) .
$$

\section{EFFECTIVE ACTION OF COLORED MASSIVE PARTICLES AND ITS DOUBLE COPY}

\section{A. Principle of the double-copy construction}

The double-copy scheme to be applied here is to first integrate out the gauge field $A_{\mu}^{a}$ in order to obtain the effective action $S_{\text {eff,YM }}$ for two colored, massive particles with Yang-Mills interactions, i.e.,

$$
e^{\frac{i}{\hbar} S_{\text {eff, YM }}}=e^{\frac{i}{\hbar} S_{\text {pc.free }}} \mathcal{M}_{\mathrm{YM}}=\text { const } \int \mathcal{D} A e^{\frac{i}{\hbar} S_{\mathrm{cYM}}}
$$

The (divergent) normalization constant on the right-hand side is chosen such that $\mathcal{M}_{\mathrm{YM}}=1$ for $g \rightarrow 0$ and $S_{\mathrm{pc} \text {,free }}$ is given by $S_{\mathrm{pc}}+\tilde{S}_{\mathrm{pc}}$ for $g \rightarrow 0$. The perturbative expansion in the coupling $g$ of $\mathcal{M}_{\mathrm{YM}}$ can always be brought into the schematic form

$$
\mathcal{M}_{\mathrm{YM}}=\sum_{n=0}^{\infty}(2 g)^{2 n} \sum_{I \in \Gamma_{n}}\left(\frac{i}{\hbar}\right)^{x_{I}} \int \prod_{i_{I}} d \hat{\tau}_{i_{I}} \int d^{4 l_{I}} x \frac{C_{I} N_{I}}{S_{I} D_{I}}
$$

where $\Gamma_{n}$ is the set of $\mathrm{N}^{n-1} \mathrm{LO}$ trivalent graphs with two external worldlines of the colored point particles, $C_{I}$ denotes the color factor associated with graph $I$, i.e., functions of the $c(\tau)$ 's, $N_{I}$ the associated kinematic numerator factors and $D_{I}$ the spacetime propagators appearing in the graph $I$. The $x_{I}$ is defined as the number of vertices (bulk and worldline) minus the number of propagators in the trivalent diagram and $l_{I}$ is the number of bulk vertices. Note that the integral measure along the worldline $d \tau$ always comes with a corresponding Lagrange multiplier $\lambda(\tau)$ in order to guarantee reparametrization invariance. We collect this into a new density notation via

$$
d \hat{\tau}_{i}:=d \tau_{i} \lambda\left(\tau_{i}\right)
$$

The $S_{I}$ finally, denote the symmetry factor of the graph $I$ in the trivalent representation, i.e., the graph has to be drawn in such a way that it reproduces the color structure $C_{I}$. We will explain our notion of a trivalent representation more precisely in the next section.

The double-copy prescription that we are adopting here then amounts to representing the exponential of the effective action of two massive particles coupled to a weak-field (post-Minkowskian) expansion of dilaton gravity (dg) as

$$
\mathcal{M}_{\mathrm{dg}}=\sum_{n=0}^{\infty}(i \kappa)^{2 n} \sum_{I \in \Gamma_{n}}\left(\frac{i}{\hbar}\right)^{x_{I}} \int \prod_{i_{I}} d \hat{\tau}_{i_{I}} \int d^{4 l_{I}} x \frac{N_{I} N_{I}}{S_{I} D_{I}},
$$

where $\kappa$ is the gravitational coupling constant. After that, the effective action in dilaton gravity follows from $e^{\frac{i}{\hbar} S_{\text {eff, dg }}}=e^{\frac{i}{\hbar} S_{\mathrm{pc} \text { free }}} \mathcal{M}_{\mathrm{dg}}$. Only classical terms $(\hbar \rightarrow 0)$ are retained in $S_{\text {eff,dg }}$ in our considerations. At higher order, $C_{I}$ and $N_{I}$ are required to fulfill the usual BCJ colorkinematics duality (Jacobi relations). We conclude this section by stressing that our double-copy prescription is proposed at the level of $\mathcal{M}$ and not at the level of the effective action. 


\section{B. The trivalent representation of the effective action of colored particles to next-to-leading order}

We now compute the effective action for two color-charged massive point particles moving on their worldlines to next-to-leading order, $\mathcal{O}\left(g^{4}\right)$. Furthermore, we will clarify what we mean by a trivalent representation of a diagram involving worldlines. We will use the short-hand notation

$$
\begin{aligned}
c_{i} & :=c\left(\tau_{i}\right), \quad p_{i}:=p\left(\tau_{i}\right), \\
D_{i j} & :=D\left(x\left(\tau_{i}\right)-x\left(\tau_{j}\right)\right), \quad d \hat{\tau}_{1 \ldots n}:=\prod_{i=1}^{n} d \tau_{i} \lambda\left(\tau_{i}\right) .
\end{aligned}
$$

Additionally, we will use the tilde notation for the "right"

$$
\tau_{2} \tau_{1} \mid=2 g^{2} \frac{i}{\hbar} \int d \hat{\tau}_{12}\left(c_{1} \cdot c_{2}\right)\left(p_{1} \cdot p_{2}\right) D_{12},
$$
worldline in our diagrams.

At leading order we have the three graphs

$$
\tau_{1} \sim \tilde{\tau}_{2}=4 g^{2} \frac{i}{\hbar} \int d \hat{\tau}_{1 \tilde{2}}\left(c_{1} \cdot \tilde{c}_{2}\right)\left(p_{1} \cdot \tilde{p}_{2}\right) D_{1 \tilde{2}}
$$

Since the last term vanishes in dimensional regularization, we neglect any graph involving a bubble, as shown above, or any other scaleless integral. Note that we do not mention the mirrored counterparts to every graph with an uneven number of untilded and tilded variables. They can be trivially obtained by replacing untilded and tilded and it is understood that we add them to our final results.

At the next-to-leading order we encounter the graphs

$$
\begin{aligned}
& \tilde{\tau}_{4} \\
& \tilde{\tau}_{2} \\
& =8 g^{4}\left(\frac{i}{\hbar}\right)^{2} \int d \hat{\tau}_{1 \tilde{2} 3 \tilde{4}}\left(c_{1} \cdot \tilde{c}_{2}\right)\left(c_{3} \cdot \tilde{c}_{4}\right)\left(p_{1} \cdot \tilde{p}_{2}\right)\left(p_{3} \cdot \tilde{p}_{4}\right) D_{1 \tilde{2}} D_{3 \tilde{4}}, \\
& =4 g^{4} \frac{i}{\hbar} \int d \hat{\tau}_{1 \tilde{2} \tilde{4} 3}\left(c_{1} \cdot \tilde{c}_{2}\right)\left(c_{3} \cdot \tilde{c}_{4}\right)\left(\tilde{p}_{2} \cdot \tilde{p}_{4}\right) D_{1 \tilde{2}} D_{3 \tilde{4}} \frac{\delta\left(\tau_{3}-\tau_{1}\right)}{\lambda_{3}},
\end{aligned}
$$

where we used a delta function in the last step in order to introduce a dummy $\tau_{3}$ integration. This can be understood as pulling apart the gluon-gluon-worldline vertex into two gluon-worldline vertices, i.e., arriving at our definition of a trivalent representation necessary for our proposed double-copy prescription (13).

The next graph is

$$
\tau_{1} \stackrel{x}{x} \overbrace{\substack{\tau_{3} \\ \tilde{\tau}_{2}}}^{\tilde{\tau}_{3}}=-4 g^{4} \frac{i}{\hbar} \int d \hat{\tau}_{1 \tilde{2} \tilde{3}} f^{a b c} c_{1}^{a} \tilde{c}_{2}^{b} \tilde{c}_{3}^{c} V_{1 \tilde{2} \tilde{3}}^{\mu \nu \rho} p_{1 \mu} \tilde{p}_{2 \nu} \tilde{p}_{3 \rho} G_{1 \tilde{2} \tilde{3}}
$$

where $V_{123}^{\mu_{1} \mu_{2} \mu_{3}}$ is the color independent part of the Yang-Mills three gluon vertex 


$$
V_{123}^{\mu_{1} \mu_{2} \mu_{3}}=\eta^{\mu_{1} \mu_{2}}\left(\partial_{1}^{\mu_{3}}-\partial_{2}^{\mu_{3}}\right)+\text { cyclic, }
$$

and $G_{1 \tilde{2} \tilde{3}}=\int d^{4} x D_{1 x} D_{\tilde{2} x} D_{\tilde{3} x}$. We proceed in an analogous fashion with

$$
\begin{aligned}
\tau_{1} \sim \tilde{\tau}_{\tilde{\tau}_{2}}^{\tilde{\tau}_{3}} & =8 g^{4} \frac{i}{\hbar} \int d \hat{\tau}_{1 \tilde{2} \tilde{3}}\left(c_{1} \cdot \tilde{c}_{2}\right)\left(\tilde{c}_{2} \cdot \tilde{c}_{3}\right)\left(p_{1} \cdot \tilde{p}_{3}\right) D_{1 \tilde{2}} D_{\tilde{2} \tilde{3}} \\
& =8 g^{4} \frac{i}{\hbar} \int d \hat{\tau}_{1 \tilde{2} \tilde{3} \tilde{4}}\left(c_{1} \cdot \tilde{c}_{2}\right)\left(\tilde{c}_{4} \cdot \tilde{c}_{3}\right)\left(p_{1} \cdot \tilde{p}_{3}\right) D_{1 \tilde{2}} D_{\tilde{4} \tilde{3}} \frac{\delta\left(\tilde{\tau}_{4}-\tilde{\tau}_{2}\right)}{\tilde{\lambda}_{4}},
\end{aligned}
$$

$$
\tau_{1} \sim \overbrace{\tilde{\tau}_{4}}^{\tilde{\tau}_{2}} \overbrace{\tilde{\tau}_{3}}^{\tilde{\tau}_{3}}=8 g^{4}\left(\frac{i}{\hbar}\right)^{2} \int d \hat{\tau}_{1 \tilde{2} \tilde{3} \tilde{4}}\left(c_{1} \cdot \tilde{c}_{2}\right)\left(\tilde{c}_{3} \cdot \tilde{c}_{4}\right)\left(p_{1} \cdot \tilde{p}_{2}\right)\left(\tilde{p}_{3} \cdot \tilde{p}_{4}\right) D_{1 \tilde{2}} D_{\tilde{3} \tilde{4}},
$$

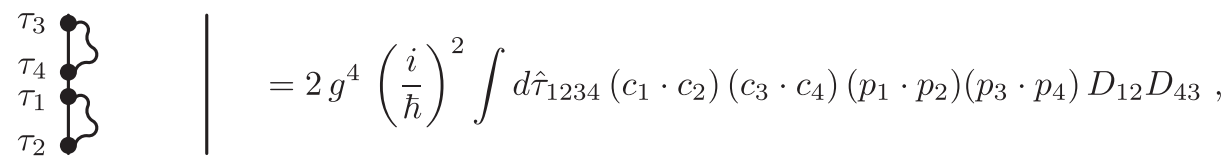

$$
\begin{aligned}
\tau_{1} \tau_{2} & =4 g^{4} \frac{i}{\hbar} \int d \hat{\tau}_{123}\left(c_{1} \cdot c_{2}\right)\left(c_{1} \cdot c_{3}\right)\left(p_{2} \cdot p_{3}\right) D_{12} D_{13} \\
& =4 g^{4} \frac{i}{\hbar} \int d \hat{\tau}_{1234}\left(c_{1} \cdot c_{2}\right)\left(c_{4} \cdot c_{3}\right)\left(p_{2} \cdot p_{3}\right) D_{12} D_{43} \frac{\delta\left(\tau_{4}-\tau_{1}\right)}{\lambda_{4}} .
\end{aligned}
$$

We also have graphs that are not mirrored counterparts of above mentioned graphs, i.e.,

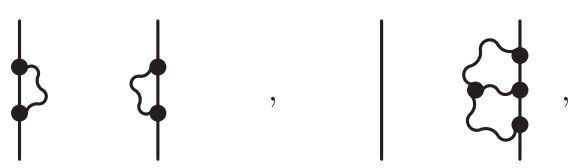

which we only include implicitly due to their very similar analytic form as previously computed graphs. In addition we neglect graphs that represent quantum corrections, since they are suppressed in the classical effective action. In particular we neglect the following type of graphs:

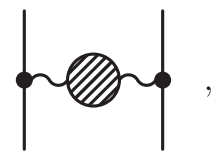

where the grey blob denotes loop topologies.

\section{The double copy of the gauge-theory effective action}

In order to perform the double copy according to (11) we first need to investigate the independent color structures from the previous section. They are given by

$$
\begin{aligned}
\mathrm{LO}: & (c \cdot \tilde{c}),(c \cdot c),(\tilde{c} \cdot \tilde{c}) . \\
\mathrm{NLO}: & (c \cdot \tilde{c})^{2},(c \cdot \tilde{c})(\tilde{c} \cdot \tilde{c}),(\tilde{c} \cdot c)(c \cdot c),(c \cdot c)^{2},(\tilde{c} \cdot \tilde{c})^{2}, \\
& f^{a b c} c_{a} c_{b} c_{c}, f^{a b c} c_{a} \tilde{c}_{b} \tilde{c}_{c}, f^{a b c} \tilde{c}_{a} c_{b} c_{c}, f^{a b c} \tilde{c}_{a} \tilde{c}_{b} \tilde{c}_{c},
\end{aligned}
$$

yielding the backbone to write down the terms in (11). We start with the $H$-graph 
$H: \tau_{1} \sim \tilde{\tau}_{2}=(2 g)^{2}\left(\frac{i}{\hbar}\right) \int d \hat{\tau}_{1 \tilde{2}} \frac{C_{H} N_{H}}{S_{H} D_{H}}$,

where

$$
\begin{aligned}
& C_{H}=\left(c_{1} \cdot \tilde{c}_{2}\right), \quad D_{H}^{-1}=D_{1 \tilde{2}}, \\
& S_{H}=1, \quad N_{H}=\left(p_{1} \cdot \tilde{p}_{2}\right) .
\end{aligned}
$$

Next we have the single-worldline (self-interaction) $D$-graph

$$
D:{ }_{\tau_{2}}^{\tau_{1}} \underset{\mid}{ } \mid=(2 g)^{2}\left(\frac{i}{\hbar}\right) \int d \hat{\tau}_{12} \frac{C_{D} N_{D}}{S_{D} D_{D}}
$$

where

$$
\begin{aligned}
& C_{D}=\left(c_{1} \cdot c_{2}\right), \quad D_{D}^{-1}=D_{12}, \\
& S_{D}=2, \quad N_{D}=\left(p_{1} \cdot p_{2}\right) .
\end{aligned}
$$

Turning to next-to-leading order contributions, we have the V-graph

Next there is the $C$-graph

$$
C: \tau_{1} \sim \overbrace{\tilde{\tau}_{4}}^{\tilde{\tau}_{2}} \overbrace{\tilde{\tau}_{2}}^{\tilde{\tau}_{3}}+\tau^{2} \overbrace{}^{\tilde{\tau}_{3}}=(2 g)^{4}\left(\frac{i}{\hbar}\right)^{2} \int d \hat{\tau}_{1 \tilde{2} \tilde{3} \tilde{4}} \frac{C_{C} N_{C}}{S_{C} D_{C}},
$$

where

$$
\begin{aligned}
C_{C} & =\left(c_{1} \cdot \tilde{c}_{2}\right)\left(\tilde{c}_{3} \cdot \tilde{c}_{4}\right), \quad D_{C}^{-1}=D_{1 \tilde{2}} D_{\tilde{3} \tilde{4}}, \quad S_{C}=2, \\
N_{C} & =\left(p_{1} \cdot \tilde{p}_{2}\right)\left(\tilde{p}_{3} \cdot \tilde{p}_{4}\right)+\frac{\hbar}{i} \frac{\delta\left(\tilde{\tau}_{2}-\tilde{\tau}_{4}\right)}{\tilde{\lambda}_{4}}\left(p_{1} \cdot \tilde{p}_{3}\right) .
\end{aligned}
$$

Note that here we use the symmetry factor of the first topology of the $C$-graph. This is consistent with our double-copy prescription which states that the correct symmetry factor is the one attributed to the trivalent graph.

Next in line is the $B$-graph

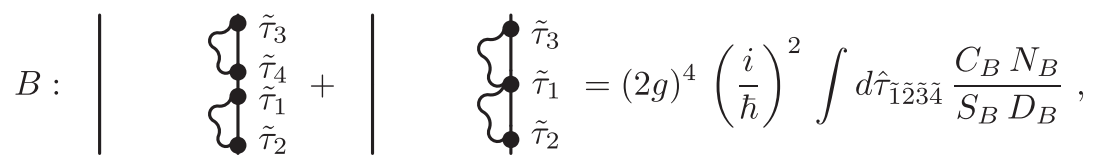


where

$$
\begin{aligned}
& C_{B}=\left(\tilde{c}_{1} \cdot \tilde{c}_{2}\right)\left(\tilde{c}_{4} \cdot \tilde{c}_{3}\right), \quad D_{B}^{-1}=D_{\tilde{1} \tilde{2}} D_{\tilde{3} \tilde{4}}, \quad S_{B}=8, \\
& N_{B}=\left(\tilde{p}_{1} \cdot \tilde{p}_{2}\right)\left(\tilde{p}_{3} \cdot \tilde{p}_{4}\right)+2 \frac{\hbar}{i} \frac{\delta\left(\tilde{\tau}_{4}-\tilde{\tau}_{1}\right)}{\tilde{\lambda}_{4}}\left(\tilde{p}_{2} \cdot \tilde{p}_{3}\right) .
\end{aligned}
$$

Again, the symmetry factor is given by the first topology of the $B$-graph.

The same constructions apply to the mirrored graphs and the graphs mentioned in (26). Performing the double copy as discussed in Sec. III A yields our prediction for the exponential of the effective action in dilaton gravity

$$
\begin{aligned}
\overline{\mathcal{M}}_{\mathrm{dg}}= & 1-\kappa^{2} \frac{i}{\hbar}\left(\int d \hat{\tau}_{H} \frac{N_{H} N_{H}}{S_{H} D_{H}}+\int d \hat{\tau}_{D} \frac{N_{D} N_{D}}{S_{D} D_{D}}\right) \\
& +\kappa^{4}\left(\frac{i}{\hbar}\right)^{2}\left(\int d \hat{\tau}_{V} \frac{N_{V} N_{V}}{S_{V} D_{V}}+\int d \hat{\tau}_{C} \frac{N_{C} N_{C}}{S_{C} D_{C}}+\int d \hat{\tau}_{B} \frac{N_{B} N_{B}}{S_{B} D_{B}}\right) \\
& +\kappa^{4} \frac{i}{\hbar} \int d \hat{\tau}_{Y} \frac{N_{Y} N_{Y}}{S_{Y} D_{Y}}+\text { (mirrored), }
\end{aligned}
$$

where we introduced the bar notation for $\overline{\mathcal{M}}_{\mathrm{dg}}$ to point out that it is computed via our double-copy prescription. At this point it is necessary to comment on a subtle feature of the above result. An essential technicality in the previous construction was the notion of pulling apart the gluon-gluon-worldline vertex using a delta function, i.e., obtaining a trivalent representation. Doing so, our double-copy prescription introduces $\delta(0)$ terms in $\overline{\mathcal{M}}_{\mathrm{dg}}$. This is a potential hazard but since such terms are of $\mathcal{O}\left(\hbar^{0}\right)$, they are quantum corrections to $S_{\text {eff,dg }}$ and we neglect them for the time being.

Another important property of $S_{\text {eff,dg }}$ is that all negative powers of $\hbar$ exponentiate, which is a priori not obvious. We check this by taking the logarithm of our double-copy result, obtaining a perturbative expansion of the effective action,

$$
\begin{aligned}
\bar{S}_{\mathrm{eff}, \mathrm{dg}}= & S_{\mathrm{pc}, \text { free }}+\frac{\hbar}{i} \log \overline{\mathcal{M}}_{\mathrm{dg}}=S_{\mathrm{pc}, \mathrm{free}}-\kappa^{2} \int d \hat{\tau}_{1 \tilde{2}}\left(p_{1} \cdot \tilde{p}_{2}\right)^{2} D_{1 \tilde{2}}-\frac{\kappa^{2}}{2} \int d \hat{\tau}_{12}\left(p_{1} \cdot p_{2}\right)^{2} D_{12} \\
& +\frac{\kappa^{4}}{2} \int d \hat{\tau}_{1 \tilde{2} \tilde{3}}\left(\frac{1}{2} V_{1 \tilde{2} \tilde{3}}^{\mu \nu \rho} p_{1 \mu} \tilde{p}_{2 \nu} \tilde{p}_{3 \rho}\right)^{2} G_{1 \tilde{2} \tilde{3}} \\
& +\frac{\kappa^{4}}{2} \int d \hat{\tau}_{1 \tilde{2} \tilde{3}}\left(p_{1} \cdot \tilde{p}_{2}\right)\left(p_{1} \cdot \tilde{p}_{3}\right)\left(\tilde{p}_{3} \cdot \tilde{p}_{2}\right) D_{1 \tilde{2}} D_{1 \tilde{3}} \\
& +\kappa^{4} \int d \hat{\tau}_{1 \tilde{2} \tilde{3}}\left(p_{1} \cdot \tilde{p}_{3}\right)\left(p_{1} \cdot \tilde{p}_{2}\right)\left(\tilde{p}_{3} \cdot \tilde{p}_{2}\right) D_{1 \tilde{2}} D_{\tilde{2} \tilde{3}} \\
& +\frac{\kappa^{4}}{2} \int d \hat{\tau}_{123}\left(p_{1} \cdot p_{2}\right)\left(p_{1} \cdot p_{3}\right)\left(p_{2} \cdot p_{3}\right) D_{12} D_{13} \\
& +(\text { mirrored })+\mathcal{O}(\hbar) .
\end{aligned}
$$

In the following we will check whether a direct construction within dilaton gravity reproduces this prediction.

\section{INTERACTION OF POINT MASSES IN DILATON GRAVITY}

In this section we are performing an analogous analysis as in the previous section for a system of two massive worldlines that are interacting in dilaton gravity.

\section{A. Dilaton gravity}

The action of dilaton gravity is given by ${ }^{1}$

\footnotetext{
${ }^{1}$ Note that the Gibbons-Hawking-York (GHY) boundary term $[45,46]$ is nonzero also in dimensional regularization for asymptotically flat spacetimes, leading to (44).
}

$$
\begin{aligned}
S_{\mathrm{dg}}= & -\frac{2}{\kappa^{2}} \int d^{4} x \sqrt{-g}\left[R-2 \partial_{\mu} \phi \partial^{\mu} \phi\right] \\
& +(\text { GHY boundary term }) \\
= & -\frac{2}{\kappa^{2}} \int d^{4} x \sqrt{-g}\left[g ^ { \mu \nu } \left(\Gamma^{\rho}{ }_{\mu \lambda} \Gamma^{\lambda}{ }_{\nu \rho}\right.\right. \\
& \left.\left.-\Gamma_{\mu \nu}^{\rho} \Gamma_{\rho \lambda}^{\lambda}\right)-2 \partial_{\mu} \phi \partial^{\mu} \phi\right],
\end{aligned}
$$

where $\kappa=m_{\mathrm{Pl}}^{-1}=\sqrt{32 \pi G}$ is the gravitational coupling (with Newton constant $G$ and Planck mass $m_{\mathrm{Pl}}$ ), $\phi$ is a real scalar field called the dilaton, $R$ is the usual Ricci scalar, $\Gamma_{\mu \nu}^{\alpha}=\left(\partial_{\mu} g_{\nu \beta}+\partial_{\nu} g_{\mu \beta}-\partial_{\beta} g_{\mu \nu}\right) g^{\alpha \beta} / 2$ is the Christoffel connection, $g_{\mu \nu}$ is the metric, and $g=\operatorname{det}\left(g_{\mu \nu}\right)$. The worldline action of a point mass (pm) coupled to gravity and a dilaton is defined by 


$$
S_{\mathrm{pm}}=-m \int d \tau e^{\phi} \sqrt{g_{\mu \nu} u^{\mu} u^{\nu}},
$$

with the worldline $x^{\mu}(\tau)$, the 4-velocity $u^{\mu}=\dot{x}^{\mu}$ and $\phi, g_{\mu \nu}$ are evaluated at $x^{\mu}(\tau)$. We again pass to the first-order version

$$
S_{\mathrm{pm}}=-\int d \tau\left(p_{\mu} u^{\mu}-\lambda\left[e^{-2 \phi} g^{\mu \nu} p_{\mu} p_{\nu}-m^{2}\right]\right) .
$$

The full action reads

$$
S_{\mathrm{dg}}+S_{\mathrm{gf}}+S_{\mathrm{pm}}+\tilde{S}_{\mathrm{pm}},
$$

with a gauge fixing part $S_{\mathrm{gf}}$ specified below. Note that we do not need an axion field here, which will be important when adding spin to the point particles [26].

\section{B. Weak-field expansion}

The weak-field approximation is defined as the expansion of the full metric around a flat Minkowski background, i.e.,

$$
\begin{gathered}
g_{\mu \nu}(x)=\eta_{\mu \nu}+\kappa h_{\mu \nu}(x), \\
g^{\mu \nu}(x)=\eta^{\mu \nu}-\kappa h^{\mu \nu}(x)+\kappa^{2} h^{\mu \lambda}(x) h_{\lambda}{ }^{\nu}(x)+\mathcal{O}\left(\kappa^{3}\right), \\
\sqrt{-g}=1+\kappa h^{\mu}{ }_{\mu}(x)-\frac{\kappa^{2}}{2}\left(h^{\mu \nu}(x) h_{\mu \nu}(x)-\left(h^{\mu}{ }_{\mu}(x)\right)^{2}\right) \\
+\mathcal{O}\left(\kappa^{3}\right),
\end{gathered}
$$

where $\kappa$ is our weak-field (or post-Minkowskian) perturbation parameter and $h_{\mu \nu}$ is the graviton. In principle we are ready to start the computation of the effective action by integrating out the graviton and dilaton field. Nevertheless, at this point one encounters large expressions during intermediate steps, e.g., the three-graviton vertex will have around 170 terms [47]. However, it is known that one can achieve a high simplification by performing field redefinitions and adding the appropriate gauge fixings [48]. Here, we aim at a field redefinition that removes the coupling of the dilaton to the worldline and also simplifies the threegraviton vertex to the square of the Yang-Mills one. Our procedure is given by:

First, choosing the following gauge fixing terms to $\mathcal{O}\left(\kappa^{2}\right)$,

$$
\begin{aligned}
S_{\mathrm{gf}}= & \frac{1}{\kappa^{2}} \int d^{4} x \sqrt{-g} f^{\mu} f_{\mu}, \\
f^{\mu}= & \Gamma_{\nu \sigma}^{\mu} g^{\nu \sigma}+\frac{\kappa^{2}}{2}\left[-\frac{1}{4}\left(\partial_{\kappa} h^{\kappa \lambda}\right) h_{\lambda^{\mu}}-\frac{1}{4}\left(\partial^{\mu} h^{\kappa \lambda}\right) h_{\kappa \lambda}\right. \\
& +\left(\partial^{\kappa} h^{\mu \lambda}\right) h_{\kappa \lambda}+\frac{3}{16}\left(\partial^{\mu} h^{\kappa}{ }_{\kappa}\right) h^{\lambda}{ }_{\lambda}-\frac{3}{8}\left(\partial^{\kappa} h^{\mu}{ }_{\kappa}\right) h^{\lambda}{ }_{\lambda} \\
& \left.-\frac{3}{8}\left(\partial^{\lambda} h^{\kappa}{ }_{k}\right) h^{\mu}{ }_{\lambda}\right],
\end{aligned}
$$

then performing the field redefinitions

$$
\begin{aligned}
h_{\mu \nu} \rightarrow & h_{\mu \nu}-\eta_{\mu \nu}\left(\frac{1}{2} h^{\mu}{ }_{\mu}+2 \phi\right) \\
& +\kappa\left(-\frac{1}{2} h_{\mu \nu} h_{\rho}^{\rho}+\frac{1}{8} \eta_{\mu \nu} h_{\rho}^{\rho} h_{\sigma}^{\sigma}+\frac{1}{2} h_{\mu \rho} h_{\nu}^{\rho}-2 \phi h_{\mu \nu}\right. \\
& \left.+2 \phi^{2} \eta_{\mu \nu}+\phi h_{\mu \nu} h_{\rho}^{\rho}\right)
\end{aligned}
$$

$$
\phi \rightarrow \phi+\frac{1}{4} h^{\mu}{ }_{\mu}
$$

and finally, adding the total derivative

$$
\begin{aligned}
0= & S_{\mathrm{TD}}=\int d^{4} x\left[\partial_{\mu}\left(\left(\partial_{\nu} h^{\mu \kappa}\right) h^{\nu}{ }_{\kappa}\right)-\partial_{\mu}\left(h^{\mu \nu}\left(\partial_{\kappa} h^{\kappa}{ }_{\nu}\right)\right)\right. \\
& +\kappa\left(\frac{1}{4} \partial_{\mu}\left(h^{\mu \nu}\left(\partial_{\nu} h^{\sigma \nu}\right) h_{\sigma \nu}\right)-\frac{1}{4} \partial_{\mu}\left(\left(\partial_{\nu} h^{\mu \lambda}\right) h_{\lambda \kappa} h^{\kappa \nu}\right)\right. \\
& \left.\left.-\frac{1}{4} \partial_{\mu}\left(h^{\mu \nu}\left(\partial_{\lambda} h_{\nu \rho}\right) h^{\rho \lambda}\right)+\frac{1}{4} \partial_{\mu}\left(h_{\nu}^{\mu} h^{\nu \lambda}\left(\partial_{\sigma} h_{\lambda}{ }^{\sigma}\right)\right)\right)\right],
\end{aligned}
$$

to the action. This procedure decouples the point masses on the worldline from the dilaton,

$$
\begin{aligned}
S_{\mathrm{pm}}= & -\int d \tau\left(p_{\mu} u^{\mu}-\lambda\left[\left(\eta^{\mu \nu}-\kappa h^{\mu \nu}+\frac{\kappa^{2}}{2} h^{\mu}{ }_{\rho} h^{\rho \nu}\right) p_{\mu} p_{\nu}\right.\right. \\
& \left.\left.-m^{2}\right]\right)+\mathcal{O}\left(\kappa^{3}\right),
\end{aligned}
$$

which means we only need to worry about the gravitondependent part of the field action for the computation of the classical effective action $S_{\text {eff,dg }}$ at next-to-leading order. Furthermore we observe a considerable simplification in the three-graviton interaction, i.e., it is given by

$$
\begin{aligned}
S_{\mathrm{dg}} & +S_{\mathrm{gf}}+S_{T D} \\
= & \int d^{4} x\left[\frac{1}{2} \partial_{\rho} h_{\mu \nu} \partial^{\rho} h^{\mu \nu}+\frac{\kappa}{4}\left(h_{\mu \nu} \partial^{\mu} \partial^{\nu} h_{\rho \sigma} h^{\rho \sigma}\right.\right. \\
& +2 h_{\mu \nu} \partial^{\sigma} h^{\mu}{ }_{\rho} \partial^{\nu} h^{\rho}{ }_{\sigma}-h_{\mu \nu} \partial^{\sigma} h_{\rho}{ }^{\mu} \partial^{\rho} h^{\nu}{ }_{\sigma}-h_{\rho \sigma} \partial^{\rho} h_{\mu \nu} \partial^{\sigma} h^{\mu \nu} \\
& \left.\left.-\partial_{\rho} \partial_{\sigma} h_{\mu \nu} h^{\rho \mu} h^{\sigma \nu}\right)\right]+\mathcal{O}\left(\kappa^{2}, \phi\right) \\
= & \int d^{4} x\left[\frac{1}{2} \partial_{\rho} h_{\mu \nu} \partial^{\rho} h^{\mu \nu}+\frac{\kappa}{4 \cdot 3 !} V_{123}^{\mu \alpha \gamma} V_{123}^{\nu \beta \delta} h_{1 \mu \nu} h_{2 \alpha \beta} h_{3 \gamma \delta}\right] \\
& +\mathcal{O}\left(\kappa^{2}, \phi\right),
\end{aligned}
$$

where $V_{123}^{\mu \alpha \gamma}$ is again the color independent part of the three gluon interaction defined by (21). We also introduced fiducial indices $1,2,3$, on $h_{\mu \nu}$ to indicate on which $h_{\mu \nu}$ 
the partial derivatives in $V_{123}^{\mu_{1} \mu_{2} \mu_{3}}$ are applied. The propagator therefore reads

$$
\left\langle h_{\mu \nu}(x) h_{\rho \sigma}(y)\right\rangle_{0}=\frac{\hbar}{i} \eta_{\mu(\rho} \eta_{\sigma) \nu} D(x-y),
$$

where the round brackets on the indices indicate a symmetrization of unit weight,

$$
\eta_{\mu(\rho} \eta_{\sigma) \nu}=\frac{1}{2}\left(\eta_{\mu \rho} \eta_{\sigma \nu}+\eta_{\mu \sigma} \eta_{\rho \nu}\right)
$$

\section{Effective action of gravitating particles to next-to-leading order}

Similar to the Yang-Mills case in Sec. III B, we compute $S_{\text {eff,dg }}$ by integrating out the graviton and scalar fields,

$e^{\frac{i}{\hbar} S_{\text {eff,dg }}}=e^{\frac{i}{\hbar} S_{\mathrm{pm}, \mathrm{free}}} \mathcal{M}_{\mathrm{dg}}=$ const $\int \mathcal{D} h \mathcal{D} \phi e^{\frac{i}{\hbar}\left(S_{\mathrm{dg}}+S_{\mathrm{gf}}+S_{\mathrm{pm}}+\tilde{S}_{\mathrm{pm}}\right)}$.
Again the (divergent) normalization constant on the right-hand side is chosen such that $\mathcal{M}_{\mathrm{dg}}=1$ for $\kappa \rightarrow 0$ and $S_{\mathrm{pm} \text {,free }}$ is given by $S_{\mathrm{pm}}+\tilde{S}_{\mathrm{pm}}$ for $\kappa \rightarrow 0$. We also neglect quantum corrections and keep all mirrored graphs implicit.

The relevant classical diagrams for $\mathcal{M}_{\mathrm{dg}}$ to order $\kappa^{4}$ read

$$
\tau_{1} \approx \Omega \tilde{\tau}_{2}=-\frac{i \kappa^{2}}{\hbar} \int d \hat{\tau}_{1 \tilde{2}}\left(p_{1} \cdot \tilde{p}_{2}\right)^{2} D_{1 \tilde{2}}
$$

$$
\tau_{1} \tau_{2} \mid=-\frac{i \kappa^{2}}{2 \hbar} \int d \hat{\tau}_{12}\left(p_{1} \cdot p_{2}\right)^{2} D_{12}
$$

$$
\tau_{1} \tau_{\tau_{2}}^{\tau_{3}}=\frac{i \kappa^{4}}{2 \hbar} \int d \hat{\tau}_{123}\left(p_{1} \cdot p_{2}\right)\left(p_{1} \cdot p_{3}\right)\left(p_{2} \cdot p_{3}\right) D_{12} D_{13},
$$




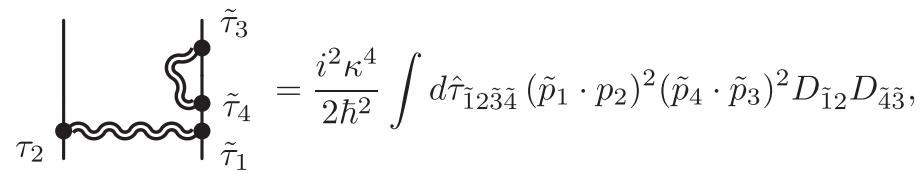

$$
\sim_{\tau_{2}}^{\overbrace{\tilde{\tau}_{1}}^{\tilde{\tau}_{3}}}=\frac{i \kappa^{4}}{\hbar} \int d \hat{\tau}_{\tilde{1} 2 \tilde{3}}\left(\tilde{p}_{1} \cdot p_{2}\right)\left(\tilde{p}_{1} \cdot \tilde{p}_{3}\right)\left(p_{2} \cdot \tilde{p}_{3}\right) D_{\tilde{1} 2} D_{\tilde{1} \tilde{3}}
$$

Summarizing, we find upon taking the logarithm that the effective action in dilaton gravity in a graphical notation reads

$$
S_{\mathrm{eff}, \mathrm{dg}}=S_{\mathrm{pm}, \text { free }}+\frac{\hbar}{i} \log \mathcal{M}_{d g}=S_{\mathrm{pm}, \text { free }}+\text { r }_{+}
$$

Now that we have obtained all classical contributions from integrating out the graviton and dilaton, we can compare this result with that from the double copy (41). Going through the expression term by term we indeed find

$$
\bar{S}_{\mathrm{eff}, \mathrm{dg}}=S_{\mathrm{eff}, \mathrm{dg}}+\int d \tau i\left(\psi^{\dagger} \dot{\psi}+\tilde{\psi}^{\dagger} \dot{\tilde{\psi}}\right)
$$

where the last term comes from the difference between $S_{\mathrm{pc} \text {,free }}$ and $S_{\mathrm{pm} \text {,free }}$. However, the dynamics of the auxiliary field $\psi$ is now decoupled and trivial such that it may be dropped. Therefore we conclude that our double-copy prescription (13) yields the correct classical effective action up to next-to-leading order in the weak-field expansion.

\section{POST-NEWTONIAN EVALUATION OF THE EFFECTIVE ACTION}

In this section, we evaluate the effective action in the post-Newtonian approximation. After adopting a gauge for the worldline parameter, the equations of motion for the Lagrange multipliers $\lambda$ and the energies $p^{0}$ of the particles become algebraic and these variables can be eliminated from the action. This leads to an action in Hamiltonian form, which agrees with previous results in scalar-tensor theory [49], that includes dilaton gravity as a special case.

The post-Newtonian approximation is a refinement of the weak-field approximation to bound binaries. The third Kepler law, or the virial theorem, then tells us that (with equality in the circular orbit case)

$$
\frac{\mathbf{v}_{r}^{2}}{c^{2}} \sim \frac{\kappa^{2}(m+\tilde{m})}{c^{2} 32 \pi r}
$$

where $r=|\mathbf{x}-\tilde{\mathbf{x}}|, \mathbf{v}_{r}$ is the relative velocity of the two particles, and we have restored the speed of light $c$. The weak-field approximation therefore implies small velocities in the case of bound binaries. It is convenient to use $c^{-1}$ as a formal post-Newtonian counting parameter, such that $\kappa=\mathcal{O}\left(c^{-1}\right)$ as well as

$$
\begin{gathered}
\left(u^{\mu}\right)=(1, \mathbf{v})=\left(\mathcal{O}\left(c^{0}\right), \mathcal{O}\left(c^{-1}\right)\right), \\
\left(p_{\mu}\right)=(E,-\mathbf{p})=\left(\mathcal{O}\left(c^{0}\right), \mathcal{O}\left(c^{-1}\right)\right),
\end{gathered}
$$

$\lambda=\mathcal{O}\left(c^{0}\right)$ and $\partial_{t}=\mathcal{O}\left(c^{-1}\right)$. The post-Newtonian expansion of the propagator then reads

$$
\begin{aligned}
D(x) & =\int \frac{d^{4} k}{(2 \pi)^{4}} \frac{e^{-i k_{\mu} x^{\mu}}}{k_{\mu} k^{\mu}+i \epsilon} \\
& =-\int \frac{d^{3} \mathbf{k}}{(2 \pi)^{3}} \frac{e^{i \mathbf{k} \cdot \mathbf{x}}}{\mathbf{k}^{2}}\left[1-\frac{\partial_{t}^{2}}{\mathbf{k}^{2}}+\frac{\partial_{t}^{4}}{\mathbf{k}^{4}}+\ldots\right] \delta(t) .
\end{aligned}
$$

Recall that the effective action is invariant under reparametrizations of the worldline parameters. We may therefore pick a gauge for them. In the post-Newtonian approximation, it is useful to fix $\tau=t=$ coordinate time. Then the effective action is the sum of the free terms $S_{\mathrm{pm} \text {,free }}$ and the connected graphs shown in (72). Most of these graphs are actually vanishing self-interactions, see Appendix B. This leaves us with (stripping off the overall factors $i / \hbar$ ) 


$$
\begin{aligned}
& \approx \approx=\frac{\kappa^{2}}{4 \pi} \int d t \frac{\lambda \tilde{\lambda} E^{2} \tilde{E}^{2}}{2 r}\left[2-4 \frac{\mathbf{p} \cdot \tilde{\mathbf{p}}}{E \tilde{E}}+\mathbf{v} \cdot \tilde{\mathbf{v}}-(\mathbf{n} \cdot \mathbf{v})(\mathbf{n} \cdot \tilde{\mathbf{v}})\right]+\mathcal{O}\left(c^{-6}\right) \\
& \overbrace{\sim}^{\sim \sim}=\frac{\kappa^{4}}{(4 \pi)^{2}} \int d t \frac{\lambda \tilde{\lambda}^{2} E^{2} \tilde{E}^{4}}{2 r^{2}}+\mathcal{O}\left(c^{-6}\right), \\
& E=m+\frac{\mathbf{p}^{2}}{2 m}-\frac{\kappa^{2} m \tilde{m}}{16 \pi r}-\frac{\mathbf{p}^{4}}{8 m^{3}}+\frac{2 \kappa^{4} m \tilde{m}(2 m+\tilde{m})}{(32 \pi r)^{2}} \\
& -\frac{\kappa^{2} m \tilde{m}}{32 \pi r}\left[\frac{\mathbf{p}^{2}}{m^{2}}+\frac{\tilde{\mathbf{p}}^{2}}{\tilde{m}^{2}}-\frac{3(\mathbf{p} \cdot \tilde{\mathbf{p}})}{m \tilde{m}}-\frac{(\mathbf{n} \cdot \mathbf{p})(\mathbf{n} \cdot \tilde{\mathbf{p}})}{m \tilde{m}}\right] \\
& +\mathcal{O}\left(c^{-6}\right) \text {, } \\
& \text { ح }
\end{aligned}
$$

where $\mathbf{n}=(\mathbf{x}-\tilde{\mathbf{x}}) / r$, and we used

$$
\int \frac{d^{d} \mathbf{k}}{(2 \pi)^{d}} \frac{e^{i \mathbf{k} \cdot \mathbf{x}}}{\left(\mathbf{k}^{2}\right)^{\alpha}}=\frac{1}{(4 \pi)^{d / 2}} \frac{\Gamma(d / 2-\alpha)}{\Gamma(\alpha)}\left(\frac{\mathbf{x}^{2}}{4}\right)^{\alpha-d / 2} .
$$

Moreover in (78) we anticipated that $\lambda=$ const + $\mathcal{O}\left(c^{-2}\right)=E$, allowing us to drop time derivatives of $\lambda, E$. The effective action in the post-Newtonian approximation finally reads

$$
\begin{aligned}
S_{\mathrm{eff}, \mathrm{dg}}= & \int d t\left[\mathbf{p} \cdot \mathbf{v}-E+\lambda\left(E^{2}-m^{2}-\mathbf{p}^{2}\right)\right. \\
& +\tilde{\mathbf{p}} \cdot \tilde{\mathbf{v}}-\tilde{E}+\tilde{\lambda}\left(\tilde{E}^{2}-\tilde{m}^{2}-\tilde{\mathbf{p}}^{2}\right) \\
& +\frac{\kappa^{2} \lambda \tilde{\lambda} E^{2} \tilde{E}^{2}}{8 \pi r}\left(2-4 \frac{\mathbf{p} \cdot \tilde{\mathbf{p}}}{E \tilde{E}}+\mathbf{v} \cdot \tilde{\mathbf{v}}-(\mathbf{n} \cdot \mathbf{v})(\mathbf{n} \cdot \tilde{\mathbf{v}})\right) \\
& \left.+\frac{\kappa^{4} \lambda \tilde{\lambda}^{2} E^{2} \tilde{E}^{4}}{2(4 \pi r)^{2}}+\frac{\kappa^{4} \tilde{\lambda} \lambda^{2} \tilde{E}^{2} E^{4}}{2(4 \pi r)^{2}}+\mathcal{O}\left(c^{-6}\right)\right] .
\end{aligned}
$$

Next, we vary the effective action with respect to $\lambda$ and $E$ to arrive at

$$
\begin{aligned}
0= & E^{2}-m^{2}-\mathbf{p}^{2} \\
& +\frac{\kappa^{2} \tilde{\lambda} E^{2} \tilde{E}^{2}}{8 \pi r}\left[2-4 \frac{\mathbf{p} \cdot \tilde{\mathbf{p}}}{E \tilde{E}}+\mathbf{v} \cdot \tilde{\mathbf{v}}-(\mathbf{n} \cdot \mathbf{v})(\mathbf{n} \cdot \tilde{\mathbf{v}})\right] \\
& +\frac{\kappa^{4}}{(4 \pi)^{2}} \frac{\tilde{\lambda}^{2} E^{2} \tilde{E}^{4}+2 \lambda \tilde{\lambda} E^{4} \tilde{E}^{2}}{2 r^{2}}+\mathcal{O}\left(c^{-6}\right), \\
0= & -1+2 \lambda E+\frac{\kappa^{2} \lambda \tilde{\lambda} E \tilde{E}^{2}}{2 \pi r}+\mathcal{O}\left(c^{-4}\right),
\end{aligned}
$$

and similar for the tilded variables. Solving iteratively for $\lambda$, $E$, using also $\mathbf{p}=m \mathbf{v}+\mathcal{O}\left(c^{-3}\right)$,

and substituting these solutions into the action, we arrive at $\left(\right.$ recall $\left.\kappa^{2}=32 \pi G\right)$

$$
S_{\mathrm{eff}, \mathrm{dg}}=\int d t[\mathbf{p} \cdot \mathbf{v}+\tilde{\mathbf{p}} \cdot \tilde{\mathbf{v}}-H]
$$

$$
\begin{aligned}
H= & m+\tilde{m}+\frac{\mathbf{p}^{2}}{2 m}+\frac{\tilde{\mathbf{p}}^{2}}{2 \tilde{m}}-\frac{2 G m \tilde{m}}{r} \\
& -\frac{\mathbf{p}^{4}}{8 m^{3}}-\frac{\tilde{\mathbf{p}}^{4}}{8 \tilde{m}^{3}}+\frac{2 G^{2} m \tilde{m}(m+\tilde{m})}{r^{2}} \\
& -\frac{G m \tilde{m}}{r}\left[\frac{\mathbf{p}^{2}}{m^{2}}+\frac{\tilde{\mathbf{p}}^{2}}{\tilde{m}^{2}}-\frac{3 \mathbf{p} \cdot \tilde{\mathbf{p}}}{m \tilde{m}}-\frac{(\mathbf{n} \cdot \mathbf{p})(\mathbf{n} \cdot \tilde{\mathbf{p}})}{m \tilde{m}}\right] \\
& +\mathcal{O}\left(c^{-6}\right) .
\end{aligned}
$$

Notice the extra factor of 2 in the Newtonian potential, which is due to the dilaton. Throughout this derivation, higher-order time derivatives (like accelerations) are removed by inserting the respective equations of motion. This corresponds to a variable redefinition at the considered order [50]. [In particular, this justifies the dropping of time derivatives of $\lambda, E$ and the use of $\mathbf{p}=m \mathbf{v}+\mathcal{O}\left(c^{-3}\right)$ above.] Further note that $H$ is the Hamiltonian describing the motion of the binary system in dilaton gravity.

We may compare our result to scalar-tensor theory [49]. Scalar-tensor theories (in the so called Einstein frame) are based on the same field action as dilaton gravity (43). However, the source part (45) is more generic in scalartensor theories,

$$
\begin{aligned}
S_{\mathrm{pm}, \mathrm{st}}= & -m \int d \tau \sqrt{g_{\mu \nu} u^{\mu} u^{\nu}} \\
& \times\left[1+\alpha_{0} \phi+\frac{1}{2}\left(\alpha_{0}^{2}+\beta_{0}\right) \phi^{2}+\mathcal{O}\left(\kappa^{3}\right)\right],
\end{aligned}
$$

with the so-called sensitivities $\alpha_{0}$ and $\beta_{0}$. Comparing to (45) we find that $\alpha_{0}=1$ and $\beta_{0}=0$ for dilaton gravity. The Hamiltonian for scalar-tensor gravity, following via a 
Legendre transform from the Lagrangian stated in Eq. (3.7) of Ref. [49], reads

$$
\begin{aligned}
H= & m+\tilde{m}+\frac{\mathbf{p}^{2}}{2 m}+\frac{\tilde{\mathbf{p}}^{2}}{2 \tilde{m}}-\frac{\tilde{G} m \tilde{m}}{r}-\frac{\mathbf{p}^{4}}{8 m^{3}}-\frac{\tilde{\mathbf{p}}^{4}}{8 \tilde{m}^{3}} \\
& -\frac{\tilde{G} m \tilde{m}}{r}\left[\frac{3 \mathbf{p}^{2}}{2 m^{2}}+\frac{3 \tilde{\mathbf{p}}^{2}}{2 \tilde{m}^{2}}-\frac{7 \mathbf{p} \cdot \tilde{\mathbf{p}}}{2 m \tilde{m}}-\frac{(\mathbf{n} \cdot \mathbf{p})(\mathbf{n} \cdot \tilde{\mathbf{p}})}{2 m \tilde{m}}\right] \\
& +\frac{2 G m \alpha_{0} \tilde{m} \tilde{\alpha}_{0}}{r}\left[\frac{\mathbf{p}}{m}-\frac{\tilde{\mathbf{p}}}{\tilde{m}}\right]^{2}+\frac{\tilde{G}^{2} m \tilde{m}}{2 r^{2}}(m+\tilde{m}) \\
& +\frac{G^{2} m \tilde{m}}{2 r^{2}}\left(m \alpha_{0}^{2} \tilde{\beta}_{0}+\tilde{m} \tilde{\alpha}_{0}^{2} \beta_{0}\right)+\mathcal{O}\left(c^{-6}\right),
\end{aligned}
$$

where $\tilde{G}=G\left(1+\alpha_{0} \tilde{\alpha}_{0}\right)$. This Hamiltonian agrees with ours for $\alpha_{0}=1$ and $\beta_{0}=0$, as expected. Note that for $\alpha_{0}=0=\beta_{0}$ one obtains the result of general relativity.

In closing let us compare the complexities of establishing the result above with the more traditional approach of Ref. [49]. At this low order in perturbation theory it is hard to claim a clear superiority of the double-copy approach. We regard our work as a proof of concept, which we expect to simplify calculations at higher orders dramatically, as it is the case in scattering amplitudes. But already at the nextto-leading order established here, our approach allows one to obtain the post-Minkowskian Feynman integrals in a simple way, where in a more traditional approach the threegraviton vertex contains around 170 terms [47].

\section{CONCLUSIONS}

In the present paper, we proposed an adaption of the $\mathrm{BCJ}$ double copy [1-3] to the classical effective action of binary systems and demonstrated its validity to next-to-leading order in the weak-field/post-Minkowskian approximation. This is another application for a BCJ-like double copy beyond (quantum) scattering amplitudes, next to classical solutions for the field equations [19-21] and weak-field approximations for the radiation from classical binary point-sources [24-26,28-30]. The former application of the double copy operates at the level of the gauge field and metric which are gauge-covariant quantities, unlike gauge-invariant scattering amplitudes. Similarly, in the present work, we formulated a double copy between effective actions, which are not manifestly gauge independent either: the actions depend on gauge-dependent canonical momenta, and on the dilaton-gravity side the positions are gauge dependent, too. ${ }^{2}$ This provides growing evidence that the BCJ double copy can be adapted to gauge-covariant quantities, which, if true, would liberate it to a considerably larger realm of applications. Furthermore, our work is an

\footnotetext{
${ }^{2}$ The (conservative) effective actions encode the binding energy (energy levels in a quantum mechanical analog), which are gauge independent. However, the variables (like momenta) that are used when the double copy is performed are not gauge invariant.
}

adaptation of the $\mathrm{BCJ}$ double copy to classical gravity. This is an important research direction due to the foreseeable improvements of gravitational wave observations over the next decade(s), demanding ever more accurate predictions for the motion and radiation of compact astrophysical binaries.

An improvable part of our double-copy procedure is the split of worldline vertices with more than one gluon, using a delta distribution of the worldline parameter. This leads to singular terms involving $\delta(0)$ in the quantum corrections to the effective action, which we boldly drop in our classical consideration. While one cannot expect a consistent treatment of all quantum corrections in an approach based on classical worldlines, addressing this problem might still lead to a crucial improvement of our approach. An interesting solution is to integrate out the auxiliary field $\psi$ along the worldline. Since its propagator is a step function, the delta distributions used to split worldline vertices can be produced by derivatives in the numerators acting on these worldline propagators. This will avoid the terms $\delta(0)$. However, after the double-copy step, this will produce an auxiliary field $\psi$ propagating along the worldline in the dilaton gravity, too. But it appears possible to surgically remove this unwanted feature from the result. Alternatively, one can possibly give the auxiliary field an additional property that one desires of the theory, like a spin. It is interesting to note that step-function propagators along the worldline basically introduce a time ordering. This time ordering is also present in the Wilson loop, which can be used to calculate the potential between a quarkantiquark pair.

We may also compare our work to a direct calculation of radiation via equations of motion in [24-26,30], which is based on the same classical color charges (Yang-Mills) and point-masses (dilaton gravity) as our work. If we descend from the effective action to the equations of motion through a variation, then derivatives of the propagators may appear. These derivatives are not part of the numerators which take part in the double copy. Adapting the double copy at the level of equations of motion then faces the challenge of identifying numerators and (derivatives of) propagators. This problem was elegantly solved by Shen [30]: replacing the Yang-Mills numerators by a copy of the color factor should lead to the corresponding result in bi-adjoint scalar theory, thus providing a prescription to separate numerator and propagator structures. Here we avoided this complication by working at the action level. On the other hand, it is straightforward to calculate the (gauge independent) radiation emitted by a binary system when working with equations of motion [24-26,30].

While maybe less straightforward, extending our work to radiation from binaries will be an interesting future direction. Note that the calculation of classical binary interaction potentials presented here was an effective field theory approach similar to [36]. Following this line of research [51], the calculation of radiation aided by a double 
copy (at the level of the action) could succeed. Past work also suggests that an extension of our work to spinning point particles [26] or a projection to pure general relativity [14,31] will be possible. But the most important future extension of our present paper will be to demonstrate the double-copy prescription at higher orders. At next-tonext-to-leading order in the effective action, the BCJ color-kinematics duality (Jacobi identity) will start to play a crucial role in identifying suitable color factors and numeraors for the double copy. As for all work in the area of color-kinematics duality and double copy, it will be highly interesting to see if our proposal (or a modification thereof) survives at the next order. For the original BCJ case, the answer to this was so far always positive.

\section{ACKNOWLEDGMENTS}

We wish to thank Alessandra Buonanno, Thibault Damour, Radu Roiban, Chia-Hsien Shen and Justin Vines for helpful discussions. The work of J. P. is supported through funds of Humboldt-University Berlin in the framework of the German excellency initiative.

\section{APPENDIX A: CONVENTIONS AND FEYNMAN RULES}

The signature of spacetime is -2 ("mostly minus"). We use units such that the speed of light $c=1$ and use $c^{-1}$ as a formal post-Newtonian counting parameter.

The Yang-Mills action is the standard one, $S_{\mathrm{YM}}=$ $-\frac{1}{4} \int d^{4} x\left(F_{\mu \nu}^{a}\right)^{2}$, with the color convention $\operatorname{tr}\left(T^{a} T^{b}\right)=$ $\delta^{a b} / 2$. The field strength $F_{\mu \nu}^{a}$ is given by

$\left[D_{\mu}, D_{\nu}\right]=-i g F_{\mu \nu}^{a} T^{a} \Rightarrow F_{\mu \nu}^{a}=\partial_{\mu} A_{\nu}^{a}-\partial_{\nu} A_{\mu}^{a}+g f^{b c a} A_{\mu}^{b} A_{\nu}^{c}$,

where $D_{\mu}=\partial_{\mu}-i g A_{\mu}^{a} T^{a}, \quad\left[T^{a}, T^{b}\right]=i f^{a b c} T^{c}$ and the structure constants $f^{a b c}$ are totally antisymmetric here. Working in Feynman gauge we have the coordinate space Feynman rules of Yang-Mills theory,

$$
\left\langle A_{\mu}^{a}(x) A_{\nu}^{b}(y)\right\rangle_{0}=a \mu \sim b \nu=\frac{\hbar}{i} \eta_{\mu \nu} \delta_{a b} D(x-y),
$$

$$
\tau \sim \sim a \mu=\frac{i}{\hbar} 2 g \lambda(\tau) p^{\mu}(\tau) c^{a}(\tau)
$$

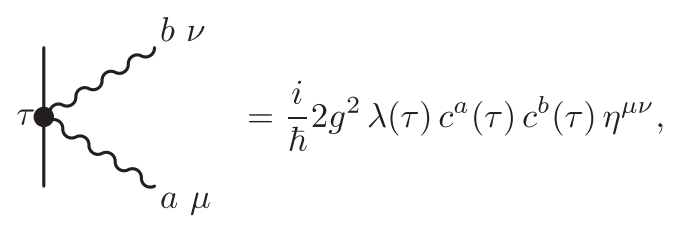

$$
\left.{ }_{b \mu_{2}}^{a \mu_{1}}\right\}^{2} c \mu_{3}=-\frac{i}{\hbar} g f^{a b c} V_{123}^{\mu_{1} \mu_{2} \mu_{3}} \text {, }
$$

where we used the abbreviation $V_{123}^{\mu_{1} \mu_{2} \mu_{3}}$ from (21).

Our definition for the Riemann tensor is $R_{\nu \alpha \beta}^{\mu}=$ $\partial_{\alpha} \Gamma_{\nu \beta}^{\mu}-\partial_{\beta} \Gamma_{\nu \alpha}^{\mu}+\Gamma_{\lambda \alpha}^{\mu} \Gamma_{\nu \beta}^{\lambda}-\Gamma_{\lambda \beta}^{\mu} \Gamma_{\nu \alpha}^{\lambda}$, from which the Ricci tensor $R_{\mu \nu}=R^{\alpha}{ }_{\mu \alpha \nu}$ and Ricci scalar $R=R^{\mu}{ }_{\mu}$ are obtained through contractions. The Feynman rules for dilaton gravity, for our choice of gauge and field redefinitions, follow straightforwardly from (59) and (56) as

$$
\left\langle g_{\mu \nu}(x) g_{\alpha \beta}(y)\right\rangle_{0}=\alpha \beta \approx \sim \mu \nu=-\frac{\hbar}{i} P_{\mu \nu \alpha \beta} D(x-y),
$$

$$
\tau \approx \approx \mu \nu=-\frac{i}{\hbar} \kappa \lambda(\tau) p^{\mu}(\tau) p^{\nu}(\tau)
$$

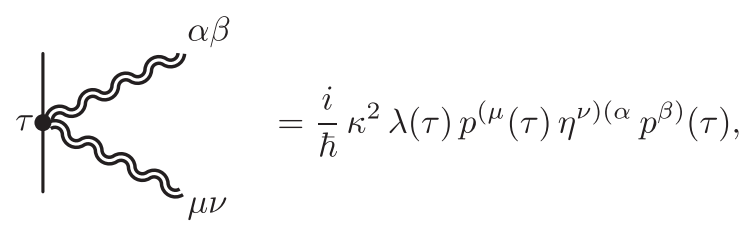

$$
\}_{\mu_{2} \nu_{2}}^{\mu_{1} \nu_{1}} \underset{\}^{2}}{2} \approx \mu_{3} \nu_{3}=\frac{i}{\hbar} \frac{\kappa}{4} V_{123}^{\alpha_{1} \alpha_{2} \alpha_{3}} V_{123}^{\beta_{1} \beta_{2} \beta_{3}} \prod_{i=1}^{3} P_{\alpha_{i} \beta_{i}}{ }^{\mu_{i} \nu_{i}} \text {, }
$$

where $P_{\mu \nu \alpha \beta}=\frac{1}{2}\left(\eta^{\mu \alpha} \eta^{\nu \beta}+\eta^{\nu \alpha} \eta^{\mu \beta}\right)$.

By comparing the Yang-Mills and dilaton-gravity Feynman rules, it is clear that the double copy of many diagrams is trivial, since the vertices already show the double-copy structure, except for the vertex (A8).

\section{APPENDIX B: SELF-INTERACTIONS IN THE POST-NEWTONIAN APPROXIMATION}

In this appendix, we wish to discuss the self-interactions, which vanish using the post-Newtonian expansion of the propagator (77). Using the gauge choice $\tau=t$, the simplest self-interaction at leading order reads

$$
\xi_{\phi}=-\frac{i \kappa^{2}}{2 \hbar} \int d t d t^{\prime}\left[p(t) \cdot p\left(t^{\prime}\right)\right]^{2} D\left(x(t)-x\left(t^{\prime}\right)\right)
$$




$$
\begin{aligned}
= & -\frac{i \kappa^{2}}{2 \hbar} \int \frac{d^{3} \mathbf{k} d t d t^{\prime}}{(2 \pi)^{3}}\left[p(t) \cdot p\left(t^{\prime}\right)\right]^{2} \frac{e^{i \mathbf{k} \cdot\left[\mathbf{x}(t)-\mathbf{x}\left(t^{\prime}\right)\right]}}{\mathbf{k}^{2}} \\
& \times\left[1+\frac{\partial_{t} \partial_{t^{\prime}}}{\mathbf{k}^{2}}+\ldots\right] \delta\left(t-t^{\prime}\right) .
\end{aligned}
$$

After performing one of the time integrations, the times are identified $t=t^{\prime}$ and in the end $e^{i \mathbf{k} \cdot\left[\mathbf{x}(t)-\mathbf{x}\left(t^{\prime}\right)\right]}=1$. The $\mathbf{k}$-integral then turns into a scaleless one and vanishes in dimensional regularization. Hence one gets a vanishing result at each post-Newtonian order. This result is physically sensible. Indeed, a crucial property of the propagator (in Minkowski signature) is that its support is on the light-cone. Now, the worldlines of massive particles can not intersect the light cones emanating from them, so that these self-interaction should be zero. Similar arguments apply to the other self-interactions at next-to-leading order in the effective action

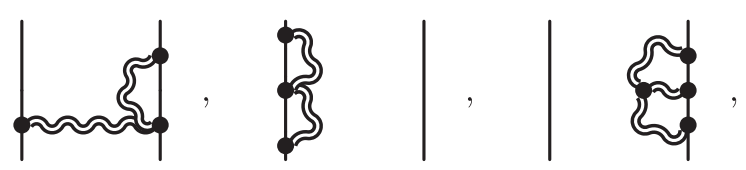

which all vanish in dimensional regularization.
[1] Z. Bern, J. J. M. Carrasco, and H. Johansson, New relations for gauge-theory amplitudes, Phys. Rev. D 78, 085011 (2008).

[2] Z. Bern, T. Dennen, Y.-t. Huang, and M. Kiermaier, Gravity as the square of gauge theory, Phys. Rev. D 82, 065003 (2010).

[3] Z. Bern, J. J. M. Carrasco, and H. Johansson, Perturbative Quantum Gravity as a Double Copy of Gauge Theory, Phys. Rev. Lett. 105, 061602 (2010).

[4] H. Kawai, D. C. Lewellen, and S. H. H. Tye, A relation between tree amplitudes of closed and open strings, Nucl. Phys. B269, 1 (1986).

[5] F. Cachazo, S. He, and E. Y. Yuan, Scattering equations and matrices: From Einstein to Yang-Mills, DBI and NLSM, J. High Energy Phys. 07 (2015) 149.

[6] Z. Bern, C. Boucher-Veronneau, and H. Johansson, $\mathcal{N} \geq 4$ supergravity amplitudes from gauge theory at one loop, Phys. Rev. D 84, 105035 (2011).

[7] Z. Bern, J. J. M. Carrasco, L. J. Dixon, H. Johansson, and R. Roiban, Simplifying multiloop integrands and ultraviolet divergences of gauge theory and gravity amplitudes, Phys. Rev. D 85, 105014 (2012).

[8] J. J. M. Carrasco, M. Chiodaroli, M. Gunaydin, and R. Roiban, One-loop four-point amplitudes in pure and mattercoupled $\mathcal{N} \leq 4$ supergravity, J. High Energy Phys. 03 (2013) 056.

[9] Z. Bern, S. Davies, T. Dennen, Y.-t. Huang, and J. Nohle, Color-kinematics duality for pure Yang-Mills and gravity at one and two loops, Phys. Rev. D 92, 045041 (2015).

[10] M. Chiodaroli, M. Gunaydin, H. Johansson, and R. Roiban, Scattering amplitudes in $\mathcal{N}=2$ Maxwell-Einstein and Yang-Mills/Einstein supergravity, J. High Energy Phys. 01 (2015) 081.

[11] M. Chiodaroli, M. Gunaydin, H. Johansson, and R. Roiban, Complete Construction of Magical, Symmetric and Homogeneous $N=2$ Supergravities as Double Copies of Gauge Theories, Phys. Rev. Lett. 117, 011603 (2016).
[12] G. Mogull and D. O'Connell, Overcoming obstacles to colour-kinematics duality at two loops, J. High Energy Phys. 12 (2015) 1.

[13] S. He, R. Monteiro, and O. Schlotterer, String-inspired BCJ numerators for one-loop MHV amplitudes, J. High Energy Phys. 01 (2016) 171.

[14] H. Johansson and A. Ochirov, Pure gravities via colorkinematics duality for fundamental matter, J. High Energy Phys. 11 (2015) 046.

[15] Z. Bern, J. J. Carrasco, W.-M. Chen, A. Edison, H. Johansson, J. Parra-Martinez, R. Roiban, and M. Zeng, Ultraviolet properties of $\mathcal{N}=8$ supergravity at five loops, Phys. Rev. D 98, 086021 (2018).

[16] A. Anastasiou, L. Borsten, M. J. Duff, M. J. Hughes, A. Marrani, S. Nagy, and M. Zoccali, Twin supergravities from Yang-Mills theory squared, Phys. Rev. D 96, 026013 (2017).

[17] A. Anastasiou, L. Borsten, M. J. Duff, A. Marrani, S. Nagy, and M. Zoccali, Are all supergravity theories Yang-Mills squared?, Nucl. Phys. B934, 606 (2018).

[18] C. Cheung, C.-H. Shen, and C. Wen, Unifying relations for scattering amplitudes, J. High Energy Phys. 02 (2018) 095.

[19] R. Monteiro, D. O'Connell, and C. D. White, Black holes and the double copy, J. High Energy Phys. 12 (2014) 056.

[20] A. Luna, R. Monteiro, D. O'Connell, and C. D. White, The classical double copy for Taub NUT spacetime, Phys. Lett. B 750, 272 (2015).

[21] A. Luna, R. Monteiro, I. Nicholson, A. Ochirov, D. O'Connell, N. Westerberg, and C. D. White, Perturbative spacetimes from Yang-Mills theory, J. High Energy Phys. 04 (2017) 069.

[22] A. Luna, R. Monteiro, I. Nicholson, D. O'Connell, and C. D. White, The double copy: Bremsstrahlung and accelerating black holes, J. High Energy Phys. 06 (2016) 023.

[23] B. P. Abbott et al. (Virgo and LIGO Scientific Collaborations), Observation of Gravitational Waves from a Binary Black Hole Merger, Phys. Rev. Lett. 116, 061102 (2016). 
[24] W. D. Goldberger and A. K. Ridgway, Radiation and the classical double copy for color charges, Phys. Rev. D 95, 125010 (2017).

[25] W. D. Goldberger and A. K. Ridgway, Bound states and the classical double copy, Phys. Rev. D 97, 085019 (2018).

[26] W. D. Goldberger, J. Li, and S. G. Prabhu, Spinning particles, axion radiation, and the classical double copy, Phys. Rev. D 97, 105018 (2018).

[27] A. P. Balachandran, P. Salomonson, B.-S. Skagerstam, and J.-O. Winnberg, Classical description of particle interacting with non-Abelian gauge field, Phys. Rev. D 15, 2308 (1977).

[28] W. D. Goldberger, S. G. Prabhu, and J. O. Thompson, Classical gluon and graviton radiation from the bi-adjoint scalar double copy, Phys. Rev. D 96, 065009 (2017).

[29] D. Chester, Radiative double copy for Einstein-Yang-Mills theory, Phys. Rev. D 97, 084025 (2018).

[30] C.-H. Shen, Gravitational Radiation from color-kinematics duality, J. High Energy Phys. 11 (2018) 162.

[31] A. Luna, I. Nicholson, D. O'Connell, and C. D. White, Inelastic black hole scattering from charged scalar amplitudes, J. High Energy Phys. 03 (2018) 044.

[32] T. Damour, P. Jaranowski, and G. Schäfer, Nonlocal-in-time action for the fourth post-Newtonian conservative dynamics of two-body systems, Phys. Rev. D 89, 064058 (2014).

[33] L. Bernard, L. Blanchet, A. Bohé, G. Faye, and S. Marsat, Energy and periastron advance of compact binaries on circular orbits at the fourth post-Newtonian order, Phys. Rev. D 95, 044026 (2017).

[34] T. Marchand, L. Bernard, L. Blanchet, and G. Faye, Ambiguity-free completion of the equations of motion of compact binary systems at the fourth post-Newtonian order, Phys. Rev. D 97, 044023 (2018).

[35] S. Foffa, P. Mastrolia, R. Sturani, and C. Sturm, Effective field theory approach to the gravitational two-body dynamics, at fourth post-Newtonian order and quintic in the Newton constant, Phys. Rev. D 95, 104009 (2017).

[36] W. D. Goldberger and I. Z. Rothstein, An effective field theory of gravity for extended objects, Phys. Rev. D 73, 104029 (2006).
[37] Y. Iwasaki, Quantum theory of gravitation vs. classical theory-fourth-order potential, Prog. Theor. Phys. 46, 1587 (1971).

[38] M. J. Duff, Quantum tree graphs and the Schwarzschild solution, Phys. Rev. D 7, 2317 (1973).

[39] B. R. Holstein and J. F. Donoghue, Classical Physics and Quantum Loops, Phys. Rev. Lett. 93, 201602 (2004).

[40] D. Neill and I. Z. Rothstein, Classical space-times from the S matrix, Nucl. Phys. B877, 177 (2013).

[41] N. E. J. Bjerrum-Bohr, J. F. Donoghue, and P. Vanhove, On-shell techniques and universal results in quantum gravity, J. High Energy Phys. 02 (2014) 111.

[42] N. E. J. Bjerrum-Bohr, P. H. Damgaard, G. Festuccia, L. Plante, and P. Vanhove, General Relativity from Scattering Amplitudes, Phys. Rev. Lett. 121, 171601 (2018).

[43] T. Damour, Gravitational scattering, post-Minkowskian approximation and effective one-body theory, Phys. Rev. D 94, 104015 (2016).

[44] T. Damour, High-energy gravitational scattering and the general relativistic two-body problem, Phys. Rev. D 97, 044038 (2018).

[45] G. W. Gibbons and S. W. Hawking, Action integrals and partition functions in quantum gravity, Phys. Rev. D 15, 2752 (1977).

[46] J. W. York, Jr., Role of Conformal Three Geometry in the Dynamics of Gravitation, Phys. Rev. Lett. 28, 1082 (1972).

[47] B. S. DeWitt, Quantum theory of gravity. 3. Applications of the covariant theory, Phys. Rev. 162, 1239 (1967).

[48] Z. Bern and A. K. Grant, Perturbative gravity from QCD amplitudes, Phys. Lett. B 457, 23 (1999).

[49] T. Damour and G. Esposito-Farese, Tensor multiscalar theories of gravitation, Classical Quantum Gravity 9, 2093 (1992).

[50] T. Damour and G. Schäfer, Redefinition of position variables and the reduction of higher order Lagrangians, J. Math. Phys. (N.Y.) 32, 127 (1991).

[51] W. D. Goldberger and A. Ross, Gravitational radiative corrections from effective field theory, Phys. Rev. D 81, 124015 (2010). 